\title{
A haptic user interface to assess the mobility of the newborn's neck
}

\author{
Said-Magomed Sadulaev \\ School of Computing Sciences \\ University of East Anglia \\ Norwich, United Kingdom \\ Said-Magamed.Sadulaev@uea.ac.uk \\ Zelimkhan Gerikhanov \\ School of Computing Sciences \\ University of East Anglia \\ Norwich, United Kingdom \\ Zelimkhan.Gerikhanov@uea.ac.uk
}

\author{
Rudy Lapeer \\ School of Computing Sciences \\ University of East Anglia \\ Norwich, United Kingdom \\ R.Lapeer@uea.ac.uk
}

\author{
Edward Morris \\ Obstetrics and Gynaecology \\ Norwich and Norfolk University Hospital \\ Norwich, United Kingdom \\ Edward.Morris@uea.ac.uk
}

\begin{abstract}
A virtual reality program has been developed to assess the strength and flexibility of a computer based model of a term fetus or newborn baby's neck. The software has a haptic/force feedback user interface which allows clinical experts to adjust the mechanical properties, including range of motion and mechanical stiffness of a newborn neck model, at runtime. The developed software was assessed by ten clinical experts in obstetrics. The empirically obtained stiffness and range of motion values corresponded well with values reported in the literature.
\end{abstract}

Keywords- Human Computer Interaction (HCI), Human Childbirth, Biomechanics, Medical Visualisation.

\section{INTRODUCTION}

The main objective of the research presented in this paper was to develop a virtual reality (VR) user interface (UI) which allows clinical experts to assess the range of motions and stiffness of a newborn baby's neck. The computer generated model of the newborn is created as part of a larger project aimed at the VR simulation of the mechanisms of childbirth during the second stage of labour. This software is called BirthView [1]. Figure 1 shows a screenshot of BirthView's UI. The purpose of BirthView is to propel a baby model through the maternal pelvis using exact physics properties, i.e. the intra-uterine pressure and maternal abdominal forces to expel the baby, and as a consequence, the (calculated) contact forces and subsequent torques on the baby's head to facilitate its passage through the birth canal. The motions or mechanisms that the baby's head performs to be successfully born are known as the cardinal movements. The objective of BirthView is to observe these cardinal movements without explicitly modelling them. The realistic behavior of the baby's neck is crucial for BirthView to exhibit all the cardinal movements. The current neck model in BirthView is approximated as a combination of longitudinal and torsional Hookean springs which have stiffness values based on the biomechanical properties of the cervical spine of postmortem human subjects (PMHS), including neonates, children, adults and animals [2-5]. These mechanical properties of the human cervical spine and its muscles have been derived from in vitro experiments. It is well known that the mechanical properties of bio-tissues rapidly alter after death due to rigor mortis. However in-vivo tests are, for obvious reasons, limited to non-destructive testing and can therefore not always assess the full range of tissue strength and sometimes not even the full range of motion (ROM). The study [2] on neonates or infants was limited to rotation in the sagittal plane, i.e. flexion and extension. Therefore, the remaining rotational values could only be derived by proportionally decreasing the reported values on adult PMHS. Moreover, no study was found which provided sufficient information on all three planes of motion to assess flexion/extension, lateral bending and lateral rotation.

The neck model in the BirthView software has therefore adopted mechanical properties of the newborn neck based on various values from the literature which have been scaled and adjusted to potentially match those of a term fetus or newborn.

To assess the full ROM, including muscle resistance (or stiffness), a VR based software with a haptic/force feedback UI, named BirthViewHaptics, was created. The aim of the software is to create a more realistic model of the mechanical behavior of the newborn baby's neck. The BirthViewHaptics software would then be used by clinical experts, i.e. midwives, obstetricians and neonatologists, to test, validate and improve the neck model currently used in the BirthView childbirth simulator [1].

\section{BACKGROUND}

Accurate biomechanical properties of a newborn's neck are of importance for a range of applications. First of all, as already outlined in the previous section, to improve the fidelity of the BirthView physics-based childbirth simulator as shown in Figure 1.

However, such data are useful in many other applications, including car crash simulations, neonatal examinations and legal applications, for example the investigation of shaken baby syndrome (SBS). 
There is a limited number of computational newborn neck models due to the paucity of pediatric biomechanical data. Not until 2012, the existing computational models, mainly from anthropomorphic testing devices (ATDs) or crash test dummies, have been utilizing either scaled adult or animal characteristics. Nevertheless, there are a few studies that have analysed the neonatal/infant spine under tensile, rotation and bending loading conditions [2-5] in neonatal and adult PMHS. In addition there is unpublished data provided by Prange and Myers via Coats and Margulies [6] through personal communication describing the bending moment of the C4-5 segment from a 24-day-old infant. However, none of these studies did consider the neck musculature, which significantly affects the resistance of the cervical spine [7]. Moreover, all studies considering a newborn cohort were limited to motion in the sagittal plane only, which corresponds to flexion/extension of the newborn head. The main reason behind omitting mechanical properties of a newborn's neck during rotation and lateral bending is due to its relative unimportance in car crash tests where only whiplash (excessive flexion/extension) is considered.

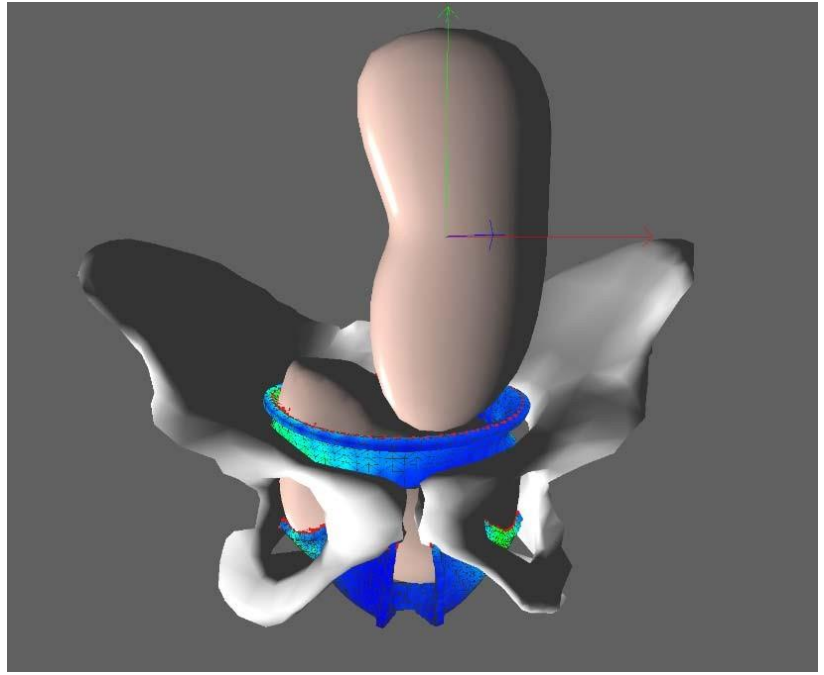

Figure 1. The BirthView physics-based childbirth simulation software. The fetal model (head, neck and trunk) is propelled through the maternal soft tissues of the bony pelvis by calculating the feto-maternal contact forces and moments as a result of the uterine and maternal abdominal expulsion forces. The figure shows the flexion of the head relative to the trunk. The realism of this cardinal movement depends on the fidelity of the fetal/newborn neck model.

Another important aspect for simulating a neck model is to identify a range of motion (ROM) of a newborn's head in all three mutually perpendicular planes (or axes), i.e. to find maximum angles of lateral rotations (twist), flexion/extension (down/up bending) and lateral bending (left/right bending). The ROM of the neck in infants, for side rotation and lateral bending, have been reported by Öhman and Beckung [8]. The ROM was measured in 38 healthy infants at the ages of 2, 4, 6 and 10 months. For lateral rotation, the mean $\mathrm{ROM}$ was $110^{\circ}$ and for lateral bending it was $70^{\circ}$.
In the remainder of this paper we will present the development and implementation of the BirthViewHaptics software. The BirthViewHaptics software was developed for the purpose of identifying the strength of a newborn's neck and its range of motion, and to find the missing biomechanical properties. The software uses two haptic devices to allow professional obstetricians and paediatricians to test and validate the newborn's neck models and obtain their expert opinion on the biofidelity of the developed models. The results of these experiments are presented in Section 4 of this paper.

\section{METHODOLOGY}

\section{A. BirthViewHaptics}

BirthViewHaptics is a custom developed simulation software, which uses two OMNI haptic devices to provide information on the effort needed to manipulate a newborn's head, visualised on a screen (see Figure 2). The left window UI allows adjustments of the mechanical properties at runtime, including the stiffness and damping coefficients of the neck. In addition, the two haptic devices allow the user to handle the virtual head with both hands to enhance realism. The virtual point of action (proxy) of the user's hands is represented by two differently coloured spheres, i.e. red for the left and yellow for the right hand.

BirthViewHaptics is using the CHAI3D haptics framework, GLFW (graphics) and the AntTweakBar (Windows UI) libraries.

CHAI3D is an open source cross-platform $\mathrm{C}++$ simulation framework for computer haptics, visualization and interactive real-time simulation. CHAI3D supports a variety of commercially available haptic devices, and makes it is easy to support new custom force feedback devices.

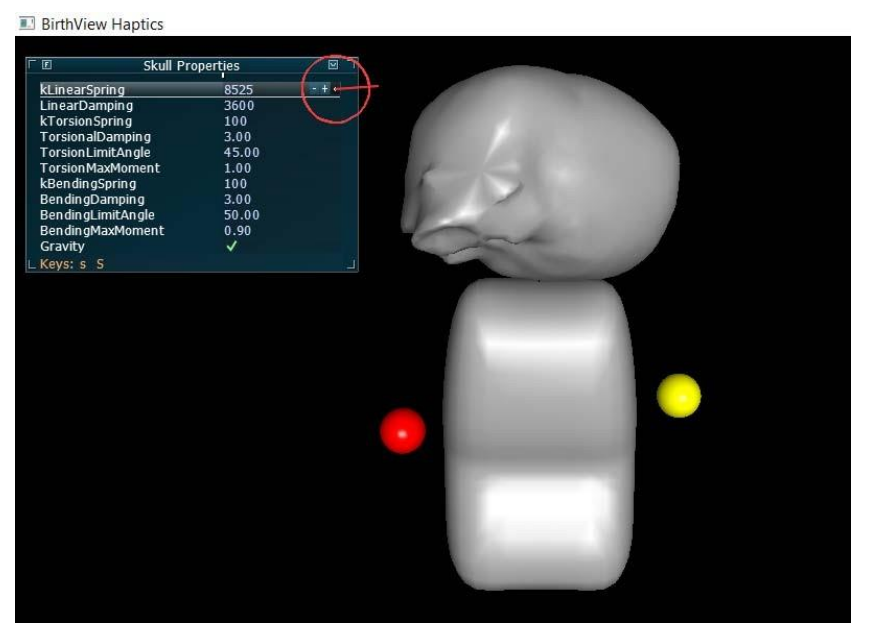

Figure 2. The BirthViewHaptics UI. The left window shows the current settings of the mechanical properties of the newborn's neck. The right window shows the model of the newborn head which is attached to the trunk via the spring-based neck model. The proxies represent the point of contact for the left hand (red) and right hand (yellow), respectively. 


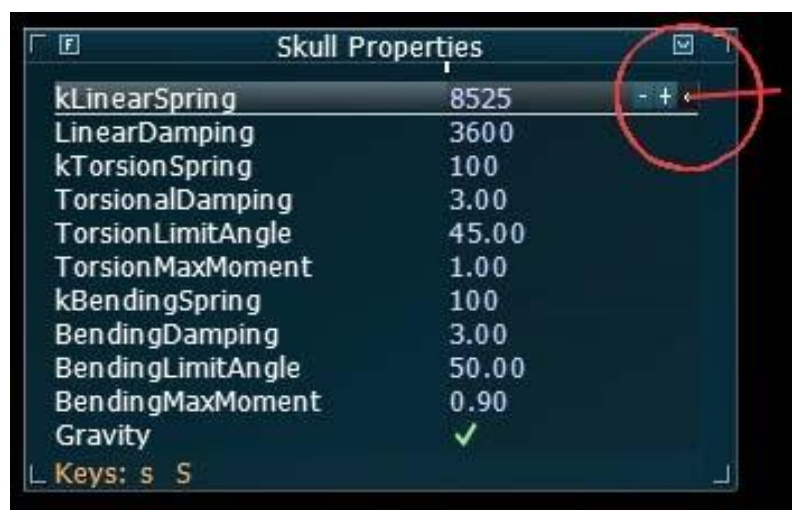

Figure 3. Enlarged view of the left window with the mechanical properties (see section 3.5. for explanation) of the newborn neck.

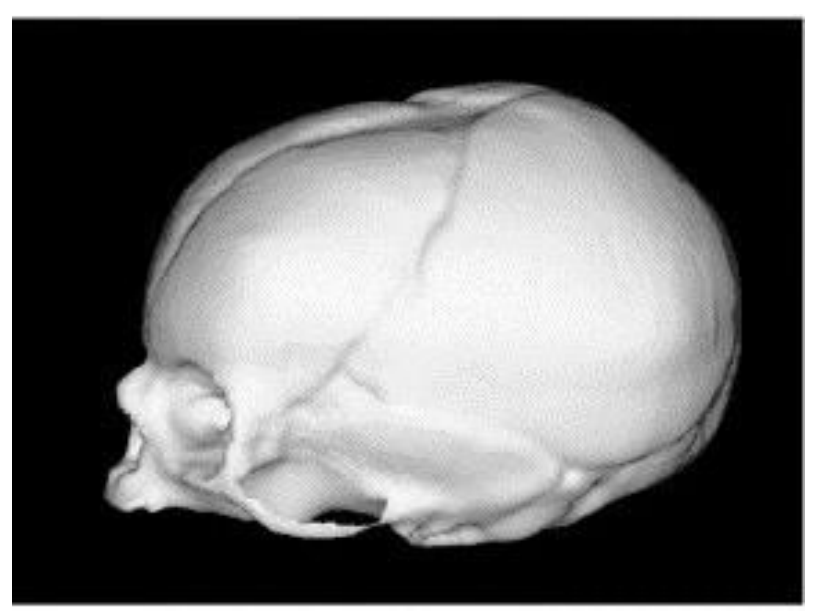

Figure 4. An initial version of the fetal head/skull model from a study on fetal head moulding [9]. The model comprises approx. $64 \mathrm{~K}$ triangular polygons.

GLFW is an open source, cross-platform library for the OpenGL API, which provides a simple API for creating windows, contexts and surfaces, receiving input and events.

AntTweakBar is a $\mathrm{C} / \mathrm{C}++$ library that allows programmers to add a graphical user interface into graphic applications based on the OpenGL API.

\section{B. Geometric models}

The newborn head model in BirthViewHaptics is a decimated newborn skull model previously used in a study on fetal head moulding [9] (see Figure 4). The polygon count has been reduced from 63,413 triangles to 2,558 triangles. The center of rotation of the skull model, i.e. pivotal point of the head is at the foramen magnum (see Figure 5).

\section{Physics simulation}

The head and torso of the virtual newborn in our software are considered to be rigid bodies and collision detection between them has not been enabled for the sake of simplicity. Both rigid bodies in the developed software can be translated across the scene and perform individual rotations. The process of finding out the object's translation can be described in the following algorithm:
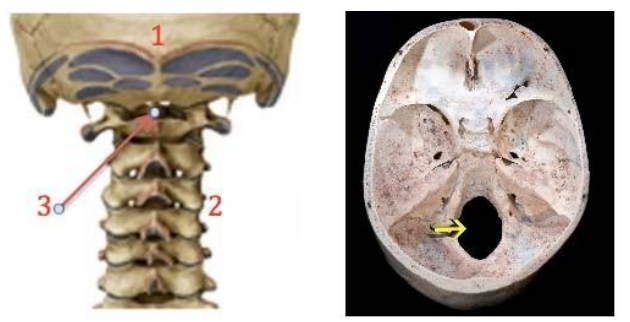

Figure 5. Foramen magnum of a human cervical spine (neck).

1. Calculate the forces acting on the object;

2. Sum up the forces in order to find a single net force;

3. Use Newton's second law to calculate the object's acceleration due to the applied forces;

$$
\boldsymbol{F}=\boldsymbol{m a}
$$

Where $\boldsymbol{m}$ is mass and $\boldsymbol{a}$ is acceleration.

4. Integrate the object's acceleration to find its velocity;

5. Integrate the object's velocity to calculate its position.

Similarly for simulating the object's rotation:

1. Calculate the torque acting on the object;

2. Add up the torques to find a single resultant torque;

3. Use Newton's second law for rotation to calculate the object's angular acceleration;

$$
\mathbf{r}=-\boldsymbol{I} \mathbf{a}
$$

Where $\boldsymbol{I}$ is moment of inertia and $\mathbf{a}$ is angular acceleration.

4. Integrate the object's acceleration to find its angular velocity;

5. Integrate the object's velocity to calculate its rotation.

Numerical integration in BirthViewHaptics uses a semi-implicit Euler method in order to integrate the object's accelerations and velocities.

Haptic interaction - CHAI3D provides a class called cGenericHapticDevice that implements a set of methods to communicate with most common 3D haptic devices. For the model of the haptic device that has been tested with BirthViewHaptics, namely the Phantom Omni, it implements a class called $c$ PhantomDevice. The force from a haptic device can be acquired by calling the method getForce(). It is then added to the resultant force and is used to calculate the torque caused by the device. Equation 3.3.3 shows the 
relationship between force, torque and moment arm, which is the distance from the pivot point to the point where the force is applied. Torque is defined as a cross product between the moment arm and the force vector.

$$
\boldsymbol{t}=\boldsymbol{r} \times \boldsymbol{F}
$$

Where $\boldsymbol{r}$ is moment arm and $\boldsymbol{F}$ is force.

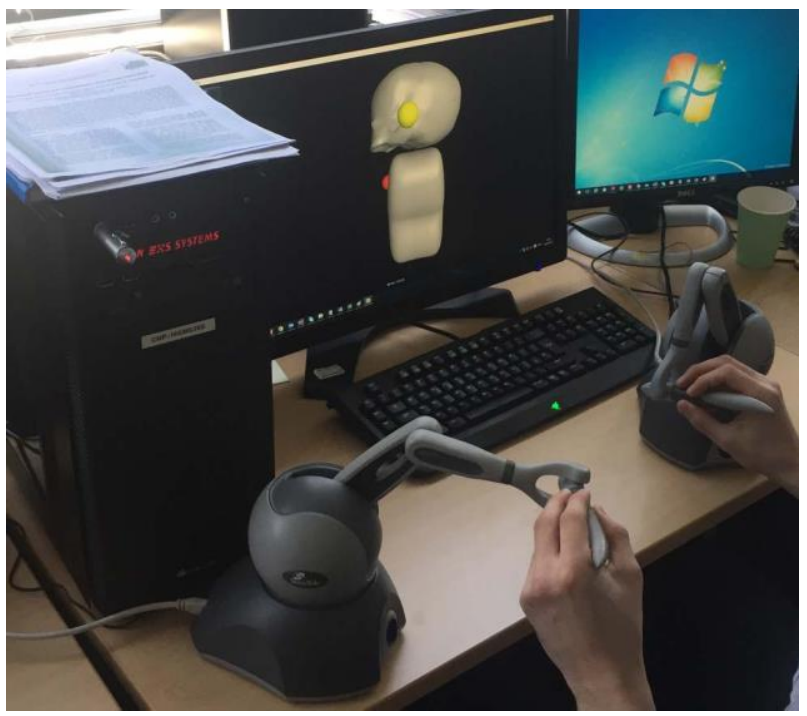

Figure 6. Figure 6. Experimental setup of the BirthViewHaptics software showing the GUI and the Phantom Omni haptics UI.

\section{Neckmodel}

The employed newborn neck model is graphically depicted as a red line segment in the GUI and is developed as a collection of spring-damper systems. The model is utilizing the stiffness parameters derived by Luck $[2,3]$. There are two types of Hookean springs in order to simulate the resistance of the virtual newborn's head. Linear springs are used for stretch/compression and torsional springs are used for bending and rotation/twist, respectively. Equation 3.4.1 is used to calculate the reacting linear force acting on the head in response to the external force from the haptic devices, i.e. the force exerted by a linear spring.

$$
F=-k x-c v
$$

Where $\boldsymbol{k}$-spring stiffness coefficient, $\boldsymbol{x}$ - displacement, $\boldsymbol{c}$ - damping coefficient, $v$ - object velocity

Equation 3.4.2 is used to calculate the reacting bending moment or torque of the head when an external force is applied to a certain point on the newborn's head.

$$
\mathbf{r}=-\kappa 8-\chi+
$$

Where $\boldsymbol{t}$ - torque, $\boldsymbol{\kappa}$-torsion coefficient, $\boldsymbol{8}$-angle of twist from an object's equilibrium position, $\chi$ - damping coefficient, + - rotational velocity.

\section{E. Neckmechanical properties}

The dimensions and masses of the fetal/newborn skull, trunk and neck were based on the data provided in the papers by Luck $[2,3]$. Hence, the length of the fetal neck was set to $3.61 \mathrm{~cm}$ (newborn aged 3 days old, identified as subject 03P [3]) and the masses of the trunk and head are 2.085 and $0.665 \mathrm{~kg}$ (newborn aged 1 day old, identified as subject $05 \mathrm{P}$ [3]) respectively. The linear damping is equal to $9650 \mathrm{Ns} / \mathrm{m}$ and $3650 \mathrm{Ns} / \mathrm{m}$ for the head and trunk respectively and the rotational damping is $3 \mathrm{Ns} /$ degree for both the head and trunk. Both of the above damping values have been obtained from BirthView [1]. The stiffness values for the tensile and bending springs are directly incorporated from Luck's data, specifically from a newborn, aged 0 days old, identified as subject 07P [2]. The tensile stiffness coefficient is equal to $7900 \mathrm{~N} / \mathrm{m} 2$ under a load displacement of $0.69 \mathrm{~mm} 2$. The flexion bending stiffness is calculated as the sum of stiffness values of the three cervical segments (O-C2, C4-C5 and C6C7) and is equal to $0.0245 \mathrm{Nm} /$ degree. The extension bending stiffness is calculated to be $0.1271 \mathrm{Nm} /$ degree. In addition, to avoid extreme bending, the stiffness values were set to increase a factor 1000 times once the maximum angle has been reached. Since we did not possess data on torsional and lateral bending stiffness, these values were set to 0 and spring constraints were introduced to stop the neck at angles specified in the studies of range of motion [8]. Thus, the range of lateral rotation and lateral bending for the neck is limited to 52.6 and 34.05 degrees respectively.

\section{EXPERIMENTS ANDRESULTS}

The developed software has been used in the experiment aimed to assess the strength and flexibility of the newborn's neck. The experiment was conducted at the Norfolk and Norwich University Hospital (NNUH) in Norwich, United Kingdom. Ten clinicians including (consultant) obstetricians, midwives and one neonatologist/pediatrician agreed to participate in the assessment and were given an induction and training session prior to the experiment to make them familiar to using the software's GUI and haptics UI.

The participants were required to apply certain forces to the newborn's head on the screen using the two haptic devices and to validate the resistance of the skull, during bending and rotation, against their real-life experience. The initial properties were then adjusted according to their feedback. The description of the procedure and instructions are given in Appendix A.

As a result, the ultimate torsional resistance (stiffness) was set to vary between 4 and $27 \mathrm{Nm}$ /degree (see Table 1) and the range of motion ranged between 57.45 and 75.06 degrees from the initial upright position. Ultimate bending stiffness ranges were between 12 and $19 \mathrm{Nm}$ /degree and the ROM for flexion was 50 degrees whereas for extension it ranged from 36.5 to 50 degrees. It is important to mention that flexion and extension were grouped together, even though in real life resistance of flexion would be noticeably less than for extension. Also lateral bending resistance and its ROM was not tested in the experiment. 
TABLE I. EXPERIMENTAL RESULTS.

\begin{tabular}{|c|c|c|c|c|c|c|c|c|c|}
\hline Clinician & RD & kT & $\chi T$ & Side Rotation & kB & $\chi \mathbf{B}$ & Flexion & Extension & Comments \\
\hline consultant & 2 & 12 & \multirow{10}{*}{3} & $70^{\circ}$ & 12 & \multirow{10}{*}{3} & $50^{\circ}$ & $50^{\circ}$ & \\
\hline obstetrician & 2 & 12 & & $70^{\circ}$ & 12 & & $50^{\circ}$ & $50^{\circ}$ & \\
\hline midwife & 2 & 7 & & $74.03^{\circ}$ & 13 & & $50^{\circ}$ & - & extension would be stiffer \\
\hline doctor/obst. & 3 & & & - & - & & - & - & looser flexion, stiffer extension \\
\hline obstetrician & 3 & 4 & & $57.45^{\circ}$ & 19 & & $50^{\circ}$ & $36.5^{\circ}$ & extension is between 36.5 and 40 degrees \\
\hline unknown & $2 / 1.5$ & 15 & & $66.22^{\circ}$ & - & & $50^{\circ}$ & $40^{\circ}$ & \\
\hline pediatrician & - & 27 & & $66.45^{\circ}$ & - & & - & - & \\
\hline doctor/obst. & 3 & 4 & & $70.43^{\circ}$ & - & & - & - & \\
\hline unknown & 2 & - & & - & - & & - & - & verified rotation only \\
\hline doctor & 3 & 8 & & $75.06^{\circ}$ & 18 & & $50^{\circ}$ & - & \\
\hline \multicolumn{10}{|c|}{$\begin{array}{l}\text { RD } \text { - rotational damping of a newborn's head (Ns/degree), } \\
\text { kT - torsion coefficient (Nm/degree), T - torsional damping coefficient (Nms/degree), } \\
\text { kB - bending coefficient (Nm/degree), B - bending damping coefficient (Nms/degree), } \\
\text { Lateral rotation, flexion and extension correspond to ROM (degrees) }\end{array}$} \\
\hline
\end{tabular}

\section{DISCUSSION}

The stiffness values for flexion and extension are much higher than the reported stiffness values in the study by Luck [2]. It was expected for them to be considerably higher since soft tissue structures can increase stiffness up to 2 times [7]. However, the calculated stiffness values are 100 and 1000 times larger for flexion and extension, respectively. This is possibly due to the fact that during our experiment only the maximum stiffness was identified at the maximum angle whereas the reported values [2] are linear approximations of an average non-linear stiffness for non-destructive bending tests.

The acquired results for the ROM compare well with the reported values of Ohman and Beckung [8] and studies by Luck [2]. Although at first glance the minimum obtained rotation ROM ranging from 57.45 degrees seems to be significantly larger than the reported 52.6 degrees it is important to note that the heads of the infants in the mentioned study were not rotated near their maximum angle so as not to harm them. However, in a virtual environment one can freely manipulate the newborn's head to the maximum possible degree without being afraid to cause damage and consequently adjust it according to what the participants believe to be its peak angle. This is one of the main advantages of using a VR GUI with haptics.

\section{CONCLUSION AND FUTURE WORK}

From the presented results it appears that the BirthViewHaptics VR simulation software is capable of providing biomechanical properties of a newborn's head motion within an acceptable margin of error, based on the assessment of professional experts, including obstetricians, midwives and neonatologists.

We successfully tested the new neck model in the BirthView simulation software. Results of these experiments are beyond the scope of this paper and will be disseminated elsewhere.

There are number of limitations to the current version of BirthViewHaptics which need to be addressed in the future:
1) Lateral bending and separate flexion from extension needs to be implemented in the software.

2) The spherical proxies should be replaced by animated hands which can grasp the fetal head for more realistic manipulations. Currently, the spherical proxies occasionally slip off the virtual head in particular with users who are not familiar with using a haptics UI.

3) More realistic 3D meshes of a newborn's head (rather than a skull model) and a trunk with shoulders and articulated arms. The legs are not important in this particular simulation.

4) The effect of gravity needs to be improved as we did not have exact data of the centre of gravity [10] of the fetal/newborn head at the time we conducted the first series of the experiments.

The above shortcomings will be addressed in the new version of BirthViewHaptics after which a new clinical experiment will be conducted to further improve the virtual newborn neck model.

\section{ACKNOWLEDGMENT}

The authors thank the staff at the NNUH in Norwich who gave up their busy schedules to participate in the experiment.

\section{REFERENCES}

[1] Gerikhanov, Z., Audinis, V. and Lapeer, R., 2013, November. Towards a forward engineered simulation of the cardinal movements of human childbirth. In E-Health and Bioengineering Conference (EHB), 2013 (pp. 1-4). IEEE.

[2] Luck, J.F., 2012. The biomechanics of the perinatal, neonatal and pediatric cervical spine: investigation (Doctoral dissertation, Duke University).

[3] Luck, J. F., Nightingale, R. W., Loyd, A. M., Prange, M. T., Dibb, A. T., Song, Y., Fronheiser, L., and Myers, B. S. (2008). Tensile mechanical properties of the perinatal and pediatric pmhs osteoligamentous cervical spine. Stapp car crash journal, 52:107.

[4] Ouyang, J., Zhu, Q., Zhao, W., Xu, Y., Chen, W., and Zhong, S. (2005). Biomechanical assessment of the pediatric cervical spine under bending and tensile loading. Spine, 30(24):E716-E723. 
[5] Nuckley, D. J., Linders, D. R., and Ching, R. P. (2013). Developmental biomechanics of the human cervical spine. Journal of biomechanics, 46(6): 1147-1154.

[6] Coats, B. and Margulies, S. S. (2008). Potential for head injuries in infants from low-height falls: Laboratory investigation. Journal of neurosurgery: pediatrics, 2(5):321-330.

[7] Duncan, J. M. (1874). Laboratory note: on the tensile strength of the fresh adult foetus. British medical journal, 2(729):763.

[8] Öhman, A. M. and Beckung, E. R. (2008). Reference values for range of motion and muscle function of the neck in infants. Pediatric Physical Therapy, 20(1):53-58.

[9] Lapeer, R.J. and Prager, R.W., 2001. Fetal head moulding: finite element analysis of a fetal skull subjected to uterine pressures during the first stage of labour. Journal of Biomechanics, 34(9), pp.11251133.

Prange, M.T., Luck, J.F., Dibb, A. and Van Ee, C.A., 2004 Mechanical properties and anthropometry of the human infant head. Stapp car crash journal, 48, p.279.

\section{APPENDIX}

Instructions to participants as to how to conduct the experiment:

Setting up stiffness and damping values

- Try to rotate the fetal head on the screen using the provided Haptics devices (See picture) and inform the experimenter if the head is resisting movement realistically
Flexion/extension/lateral bending testing

1. Try to bend the fetal head and inform the experimenter if the head is resisting movement realistically

2. Allow time to adjust the stiffness of the neck if necessary

3. Bend until the head completely resists further bending and inform the experimenter about the realism of the maximum bending angle

4. Try to flex further and inform the experimenter whether resistance of the head feels realistic.

Side rotations testing

1. Try to rotate the fetal head and inform the experimenter if the head is resisting rotation realistically

2. Allow time to adjust the stiffness of the neck if necessary

3. Rotate until the head completely resists further rotation and inform the experimenter about the realism of the maximum rotational angle

4. Try to rotate further and inform the experimenter whether resistance of the head feels realistic. 